\title{
Unmeasurable small size of foveal avascular zone without visual impairment in optical coherence tomography angiography
}

\author{
Tatsuro Yokoyama ${ }^{1} \cdot$ Ichiro Maruko $^{1} \cdot$ Hideki Koizumi $^{1,2} \cdot$ Yutaka Ishikawa $^{1} \cdot$ Tomohiro lida $^{1}$ \\ Received: 29 July 2017 / Revised: 27 October 2017 / Accepted: 14 November 2017 / Published online: 5 February 2018 \\ (c) The Royal College of Ophthalmologists 2018
}

\begin{abstract}
Purpose To report the clinical characteristics of eyes with an unmeasurable small size of foveal avascular zone (FAZ) in the optical coherence tomography angiographic (OCTA) images.

Methods Two-hundred sixty-seven eyes of 255 patients (mean age 60.4 years) without retinal and choroidal disorders to cause any type of visual impairment were examined by optical coherence tomography angiography (OCTA; RTVue XR Avanti, Optovue, Fremont, CA). Cross-sectional images at the fovea (DRI-OCT, Topcon, Japan) and fundus autofluorescence (CX-1 MYD/NM, Canon, Japan) were also recorded from all eyes.

Results Four eyes (1.5\%) of 3 patients ( 2 men, 1 woman; average age, 63.3 years) were found to have an unmeasurable small size of FAZ in the OCTA images. The best-corrected visual acuity was better than 20/20 in all eyes. Cross-sectional OCT images showed the presence of a foveal depression and the inner retinal layers in the foveal depression. These inner retinal layers were detected as a hyperreflective bands at the fovea. Fundus autofluorescence showed hypo-autofluorescence at the fovea as in normal eyes.

Conclusions An unmeasurable small size of FAZ without visual impairment was detected in $1.5 \%$ of 267 normal eyes. These eyes may be classified as low-grade foveal hypoplasia.
\end{abstract}

\section{Introduction}

The formation of the foveal depression in normal eyes results from the displacement of the inner retinal layers from the center of the fovea at around 25 weeks of gestation $[1,2]$. At the same time, a foveal avascular zone (FAZ) is formed by the suppression of retinal angiogenesis at the foveal area [1,2]. A congenital failure of these developmental steps can result in foveal hypoplasia and a reduction in the visual acuity. Foveal hypoplasia can be classified into 4 types according to the foveal shapes in the optical coherence tomographic (OCT) images. This classification was based on the presence or absence of a foveal depression [3]. In another study, optical densitometry of the fundus

Ichiro Maruko

imaruko@twmu.ac.jp

1 Department of Ophthalmology, Tokyo Women's Medical University, Tokyo, Japan

2 Department of Ophthalmology, University of the Ryukyus, Nishihara, Japan autofluorescence with a dual-wavelength system showed a reduction of the macular pigment in eyes with foveal hypoplasia [4].

Optical coherence tomography angiography (OCTA) is a relatively new technique that can record the retinal blood vessel patterns in en face images [5, 6]. It can obtain highresolution en face images of the fundus vasculatures, but it cannot detect the leakage of fluid as with fluorescein angiography. Earlier studies have shown that the mean FAZ area is about $0.25-0.30 \mathrm{~mm}^{2}$ in normal eyes [7-9]. It was recently reported that a FAZ was not present in eyes with low-grade foveal hypoplasia $[10,11]$.

The purpose of this study was to evaluate the clinical characteristics and the incidence of an unmeasurable small size of FAZ in the OCTA en face images of normal eyes.

\section{Materials and methods}

The medical records of 267 eyes of 255 consecutive individuals (mean age 60.4 years) without retinal and choroidal disorders that can cause a visual impairment were studied. All if the individuals were examined from March to 


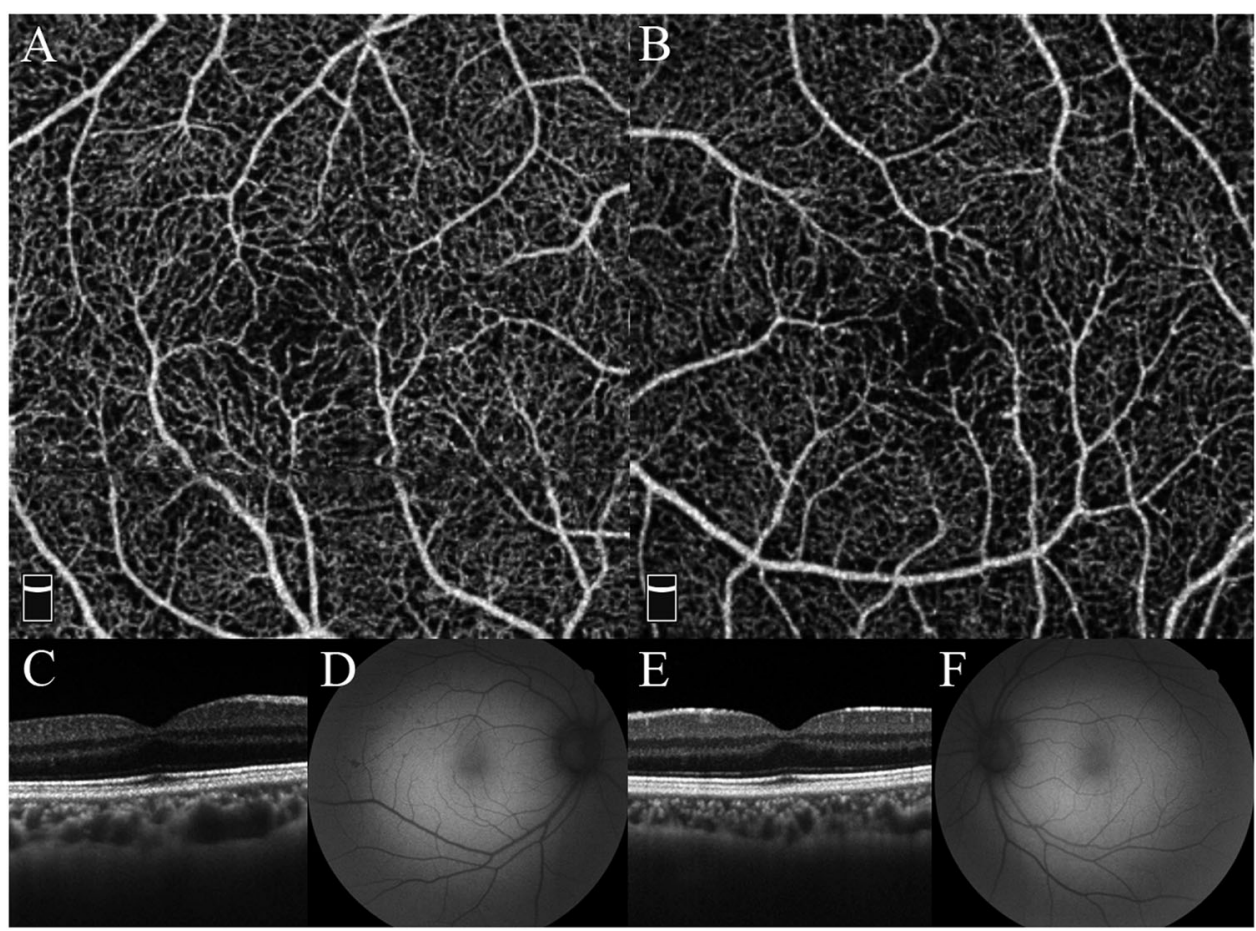

Fig. 1 Case 1 was a 37-year-old man with normal vision and normal foveal pit. a Optical coherence tomography angiography (OCTA) shows an unmeasurable small foveal avascular zone (FAZ) in the right eye. b Cross-sectional optical coherence tomography (OCT) shows an inner retinal layer, which is seen as a hyperreflective band in spite of the existence of the foveal depression in the right eye. c Fundus

December 2015 at the Tokyo Women's Medical University Hospital specializing in retinal diseases. We examined these eyes with the RTVue XR Avanti, an OCTA instrument (Optovue, Fremont, CA). The RTVue XR Avanti can detect the microvascular structures using the algorithm of splitspectrum amplitude-decorrelation angiography. This system obtains 70,000 A-scans/second with motion correction technology which helps developing the OCTA $3 \times 3 \mathrm{~mm}$ en face images. The contained software in the RTVue XR Avanti automatically shows four en face OCTA images at four depths to show the superficial capillary plexus, deep capillary plexus, outer retina, and choriocapillaris layers. In the cases of an unmeasurable small size of FAZ in the auto segmentation, the entire retina was manually segmented and re-evaluated because auto segmentation often resulted in segmentation errors [12]. Cross-sectional OCT images were obtained with a swept source OCT (DRI-OCT, Topcon, Japan), and the fundus autofluorescence was recorded with a high-resolution fundus camera (CX-1 MYD/NM, Canon, Japan).

This was a retrospective study and the procedures conformed to the tenets of the Declaration of Helsinki. The Institutional Review Board of the Tokyo Women's Medical autofluorescence shows normal hypo-autofluorescence at the fovea in the right eye. d OCTA image shows the absence of a FAZ in the left eye. e Cross-sectional OCT shows an inner retinal layer, which is seen as a hyperreflective band in spite of the existence of the foveal depression in the left eye. f Fundus autofluorescence shows normal hypo-autofluorescence at the fovea in the left eye

University, School of Medicine approved this study. This study consisted of the OCT, OCTA, and autofluorescence observations of eyes with age-related macular degeneration, with macular and retinal disorders, and normal eyes. All examinations were measured after informed consent was obtained.

\section{Results}

Among the 267 eyes of 255 patients, four eyes (1.5\%) of 3 patients ( 2 men, 1 woman; average age, 63.3 years) were found to have an unmeasurable small size of FAZ. The bestcorrected visual acuity was better than 20/20 in these 4 eyes. The medical records showed that there were no abnormalities during the fetal and perinatal periods in all 3 cases. Analyses by OCTA with manual segmentation adjusted to include the entire inner retinal layer showed retinal capillary vessels that passed through the center of the fovea. The vessels existed as a single-layer of capillaries in the foveal area. There were no other vascular abnormalities in the outer retina and the choroidal capillary slab in these eyes. 
Cross-sectional OCT images showed a thin hyperreflective band that passed through the foveal depression at the fovea. This hyperreflective band represented more than one layer of retinal cells. The fundus autofluorescence images showed normal hypo-autofluorescence at the fovea in all eyes although the images in one eye showed granular hyper-autofluorescence due to drusen.

\section{Case presentations}

Case 1 was a 37-year-old man who reported floaters in both eyes, and his BCVA was 20/20 in both eyes. Ophthalmoscopy showed a normal appearing fundus. OCTA showed retinal capillary vessels crossing the center of the fovea in both eyes (Fig. 1a-d). Cross-sectional OCT images showed a residual inner retinal layer that was seen as a hyperreflective band in the OCT images. This band passed across the foveal depression in both eyes (Fig. 1b-e). The posterior vitreous was not detached from the retina. Fundus autofluorescence showed normal hypo-autofluorescence at the fovea in both eyes (Fig. 1c-f).

Case 2 was a 74-year-old woman with cystoid macular edema in her left eye due to a branch retinal vein occlusion (BRVO). She visited our hospital every 2 or 3 months for an intravitreal injection of anti-vascular endothelial growth factor (VEGF) to treat the left eye. Ophthalmoscopy showed that the fundus of the right eye appeared normal. OCTA showed retinal capillary vessels crossing the center of the fovea (Fig. 2a). Cross-sectional OCT images showed a residual inner retinal layer despite the existence of a foveal depression (Fig. 2b). Fundus autofluorescence revealed the normal hypo-autofluorescence at the fovea (Fig. 2c).

Case 3 was a 79-year-old man who visited our hospital for age-related macular degeneration in the left eye. He did not have any visual impairments in his right eye, and ophthalmoscopy showed an almost normal appearing fundus in the right eye except for drusen, but OCTA showed retinal capillary vessels running across the center of the fovea (Fig. 3a). Cross-sectional OCT images showed a protrusion of the retinal pigment epithelium, indicating the presence of soft drusen. The inner retinal layers were present in spite of the existence of a foveal depression (Fig. 3b). Fundus autofluorescence revealed the hypo- and hyper-autofluorescence due to drusen (Fig. 3c).

\section{Discussion}

Our result showed an unmeasurable small size of FAZ in 4 of 267 eyes $(1.5 \%)$. None of these 4 eyes had any visual impairments. The inner retinal hyperreflective band was present at the fovea in the cross-sectional OCT images in

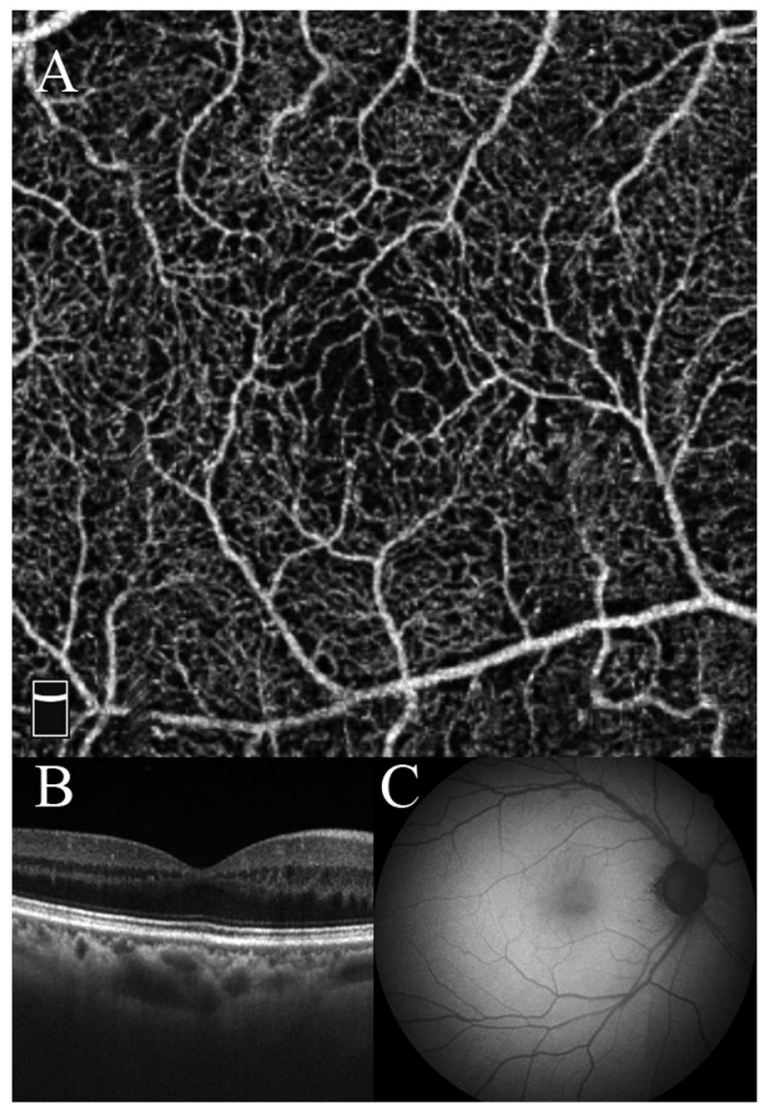

Fig. 2 Case 2 was a 74-year-old woman. a OCTA shows an unmeasurable small size of FAZ. b Cross-sectional OCT image shows the inner retinal layers in spite of the existence of a foveal depression. c Fundus autofluorescence shows normal hypo-autofluorescence at the fovea

spite of a presence of a foveal depression in all eyes. This band that ran across the fovea probably represented the inner retinal layers. These cases might be classified as eyes with low-grade foveal hypoplasia.

Foveal hypoplasia is a congenital anomaly caused by a failure in the development of a normal foveal depression and is present in eyes with albinism, congenital aniridia, and PAX6 mutations. It can also occur in isolated cases without any other ocular abnormalities [13, 14]. Thomas et al. [3] studied 69 cases of foveal hypoplasia which they graded into 4 groups according to the shape of the fovea in the OCT images. The grades were based on the presence or absence of a foveal depression, a widening of the outer nuclear layer, and the presence of photoreceptor outer segments. They reported that grade 1 eyes had foveal depressions and had the best vision, and grade 4 eyes did not have a foveal depression and had the worst vision. Because our cases had good vision with foveal depression, these could be classified as grade 1 foveal hypoplasia. 


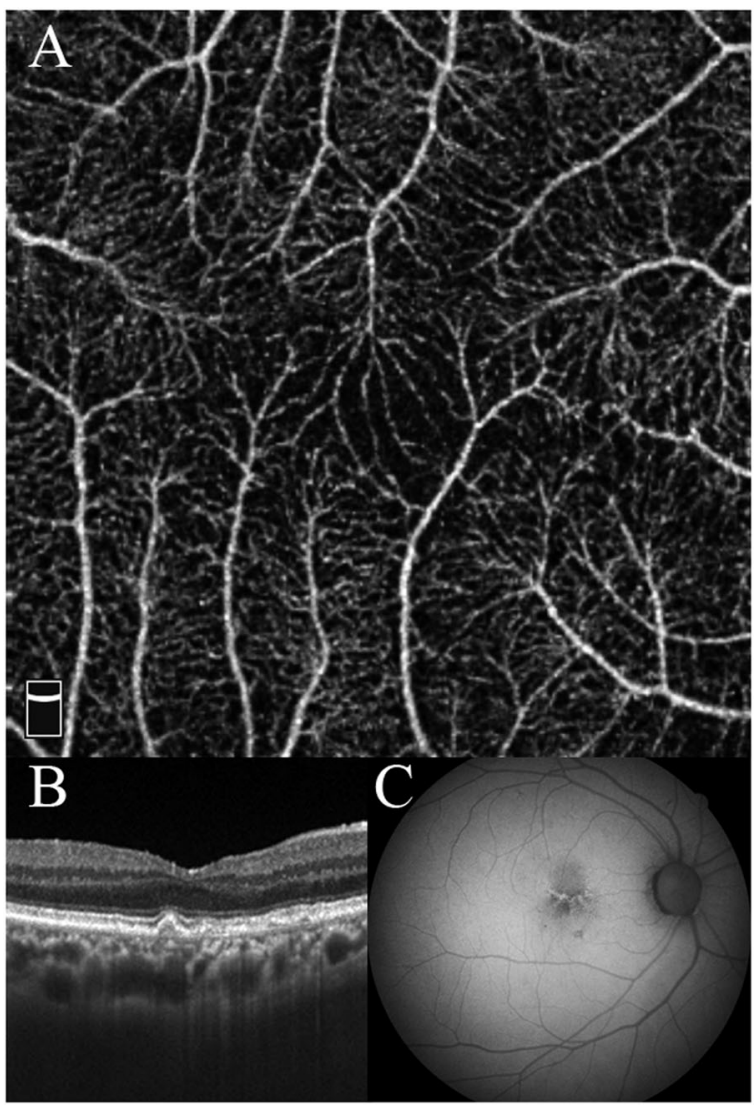

Fig. 3 Case 3 was a 79-year-old man. a OCTA shows an unmeasurable small size of FAZ. b Cross-sectional OCT image shows a protrusion of the retinal pigment epithelium indicating the presence of soft drusen. A hyperreflective band due to residual inner retinal layers is present. c Fundus autofluorescence shows the linear hyperautofluorescence due to drusen and normal hypo-autofluorescence at the fovea

The development of the fovea begins with a retina with all retinal layers present. Then, the cells of the inner retinal layers including the retinal ganglion cells migrate away from the fovea or undergo apoptosis at around 25 weeks of gestation as the second step. The foveal depression finally forms accompanied by the disappearance of the inner retinal layers $[1,2]$. During these processes, the retinal vessels are believed to not to invade the foveal region which remains avascular. However, the retinal vessels might invade to the foveal area because our cases had the residual inner retinal layers despite of the presence of the foveal depression.

Wolfson et al. [4] reported that eyes with albinism did not have a foveal depression and had low levels of macular pigment. Generally, the macular pigments are mostly distributed in the outer plexiform layer at the fovea and especially in Henle's fiber layer. Fundus autofluorescence normally shows hypo-autoflurescence due to the macular pigment. In our cases, the abnormal findings were not detected in all eyes excluding an eye with drusen. This might indicate that the macular pigment distribution was almost normal. It might be reasonable for these eyes to have good vision in spite of the unmeasurable small size of FAZ. However, further studies of the macular pigment distribution are needed to measure the macular pigment density accurately with a device with a dual-wavelength system.

Bazvand et al. [10] and Pakzad-Vaezi et al. [11] reported cases of foveal hypoplasia with small or no FAZ using OCTA as in our cases. A foveal depression as low-grade foveal hypoplasia was present in 2 of 3 cases and 2 of 6 cases, respectively. They did not report the incidence of the eyes without a FAZ in normal eyes. Although Carpineto et al. [9] reported a case without a FAZ as an example case of exclusion in their article about the reproducibility and repeatability of FAZ measurements using OCTA for 60 eyes of healthy volunteers, they never mentioned the actual number or incidence. To the best of our knowledge, our study is the first report on the incidence of eyes with unmeasurable small size of FAZ among normal eyes.

There are several weaknesses in our study, including one eye with drusen, the small bilateral cases because our hospitals specialize in retinal diseases, and no data for precise macular pigment. Although we do not know whether the very small FAZ is a distinct abnormality or a mild anomaly, we should keep in mind the existence of eyes with unmeasurable small size of FAZ. A regular ophthalmologic examination, including OCTA would be necessary for these cases. Because OCTA can determine the presence of FAZ noninvasively, it is helpful to research of the FAZ formation and foveal hypoplasia more than ever.

\section{Summary}

\section{What was known before}

- OCTA can provide information on the presence of a normal or abnormal size of FAZ in normal and diseased eyes.

- Foveal hypoplasia can be classified into 4 types according to the foveal shapes in the OCT images.

\section{What this study adds}

- OCTA showed that the FAZ was too small to measure in 4 of 267 normal eyes of 255 patients even though a normal foveal depression and macular pigment were present and the vision was good.

Acknowledgements The authors thank Professor Emeritus Duco Hamasaki of the Bascom Palmer Eye Institute, University of Miami, for his critical discussion and editing of the final manuscript. 
Author Contributions Designing and conducting of the study (TY, IM, HK); collection (TY, IM, HK, YI); management, analysis, and interpretation of the data (TY, IM, HK, YI); preparation and review (TY, IM, TI); and approval of the manuscript (TY, IM, HK, YI, TI).

\section{Compliance with ethical standards}

Conflict of interest IM reports grants from JSPS KAKENHI (Grant Number JP16K11274), grants and personal fees from Novartis Pharma K.K., personal fees from Bayer Yakuhin, Ltd., personal fees from Santen Pharmaceutical Inc., personal fees from Alcon Japan, Ltd., personal fees from Topcon Co., Ltd., personal fees from Senju Pharmaceutical Co., Ltd., personal fees from NIDEK Co., Ltd., outside the submitted work. HK reports grants and personal fees from Novartis Pharma K.K., personal fees from Bayer Yakuhin, Ltd., personal fees from Santen Pharmaceutical Inc., personal fees from Alcon Japan, Ltd., personal fees from Topcon Co., Ltd., personal fees from Senju Pharmaceutical Co., Ltd., personal fees from HOYA Corp., personal fees from Canon Inc., personal fees from Wakamoto Pharmaceutical Co., Ltd., personal fees from NIDEK Co., Ltd., outside the submitted work. TI reports grants and personal fees from Novartis Pharma K.K. (Japan), personal fees from Bayer Yakuhin, Ltd. (Japan), grants and personal fees from Santen Pharmaceutical Co., Ltd. (japan), grants from Nidek, outside the submitted work. The remaining authors declare that they have no competing interests.

\section{References}

1. Hendrickson AE, Yuodelis C. The morphological development of the human fovea. Ophthalmology. 1984;91:603-12.

2. Provis JM. Development of the primate retinal vasculature. Prog Retin Eye Res. 2001;20:799-821.

3. Thomas MG, Kumar A, Mohammad S, Proudlock FA, Engle EC, Andrews C, Chan WM, Thomas S, Gottlob I. Structural grading of foveal hypoplasia using spectral-domain optical coherence tomography a predictor of visual acuity? Ophthalmology. 2011;118:1653-60.
4. Wolfson Y, Fletcher E, Strauss RW, Scholl HP. Evidence of macular pigment in the central macula in albinism. Exp Eye Res. 2016;145:468-71.

5. Jia Y, Tan O, Tokayer J, et al. Split-spectrum amplitude-decorrelation angiography with optical coherence tomography. Opt Express. 2012;20:4710-25.

6. Spaide RF, Klancnik JM Jr, Cooney MJ. Retinal vascular layers imaged by fluorescein angiography and optical coherence tomography angiography. JAMA Ophthalmol. 2015;133:45-50.

7. Takase N, Nozaki M, Kato A, Ozeki H, Yoshida M, Ogura Y. Enlargement of foveal avascular zone in diabetic eyes evaluated by en face optical coherence tomography angiography. Retina. 2015;35:2377-83.

8. Samara WA, Say EA, Khoo CT, Higgins TP, Magrath G, Ferenczy S, Shields CL. Correlation of foveal avascular zone size with foveal morphology in normal eyes using optical coherence tomography angiography. Retina. 2015;35:2188-95.

9. Carpineto P, Mastropasqua R, Marchini G, Toto L, Di Nicola M, Di Antonio L. Reproducibility and repeatability of foveal avascular zone measurements in healthy subjects by optical coherence tomography angiography. $\mathrm{Br} \quad \mathrm{J}$ Ophthalmol. 2016;100:671-6.

10. Pakzad-Vaezi K, Keane PA, Cardoso JN, Egan C, Tufail A. Optical coherence tomography angiography of foveal hypoplasia. Br J Ophthalmol. 2017;101:985-8.

11. Bazvand F, Karkhaneh R, Roohipoor R, Rajabi MB, Ebrahimiadib N, Davoudi S, Modjtahedi BS. Optical Coherence tomography angiography in foveal hypoplasia. Ophthalmic Surg Lasers Imaging Retina. 2016;47:1127-31.

12. Spaide RF, Curcio CA. Evaluation of segmentation of the superficial and deep vascular layers of the retina by optical coherence tomography angiography instruments in normal eyes. JAMA Ophthalmol. 2017;135:259-62.

13. Azuma N, Nishina S, Yanagisawa H, Okuyama T, Yamada M. PAX6 missense mutation in isolated foveal hypoplasia. Nat Genet. 1996;13:141-2.

14. Karaca EE, Çubuk MÖ, Ekici F, Akçam HT, Waisbourd M, Hasanreisoğlu M. Isolated foveal hypoplasia: clinical presentation and imaging findings. Optom Vis Sci. 2014;91:S61-5. 